\title{
Solitary plasmacytoma in the maxillary sinus: 10-year follow-up
}

\author{
Truc Thi Hoang Nguyen*, Mi Young Eo*, Buyanbileg Sodnom-Ish, Yun Ju Cho, Soung Min Kim \\ Department of Oral and Maxillofacial Surgery, Dental Research Institute, School of Dentistry, Seoul National University, Seoul, Korea
}

\begin{abstract}
J Korean Assoc Oral Maxillofac Surg 2021;47:471-475)
Solitary plasmacytoma (SP) is an uncommon form of plasma cell neoplasm occurring intraosseously or in soft tissue and presents as a single mass of monoclonal plasma cells. SP in the maxillary sinus is rare and can be misdiagnosed as other maxillary sinus tumors. The essential examinations in patients with the initial diagnosis of plasmacytoma are bone marrow biopsy, serum and urine electrophoresis, and kappa/lambda ratio ( $\kappa: \lambda$ ratio) to rule out multiple myeloma (MM). Herein, a rare case of SP in the maxillary sinus treated by surgery and localized radiation is reported. At the 10-year follow-up examination, local recurrence or disseminated development of MM were not evident.
\end{abstract}

Key words: Plasmacytoma, Maxillary sinus, Multiple myeloma

[paper submitted 2019. 9. 15 / revised 2019. 10. 29 / accepted 2019. 11. 6]

\section{Introduction}

Plasma cell neoplasms are malignant immunosecretory disorders characterized by the monoclonal proliferation of plasma cells. Solitary plasmacytomas (SPs) are localized plasma cell neoplasms that can originate either from bone marrow plasma cells, referred to as solitary bone plasmacytoma (SBP), or soft tissue plasma cells, defined as solitary extramedullary plasmacytoma (SEP). Both SBPs and SEPs occur in patients ranging from 50 to 80 years of age and a median age of 55 years ${ }^{1,2}$. SBP occurs in males and females at a ratio of $2: 1$ and SEP occurs at a 3:1 ratio.

Recommended diagnostic criteria for SP according to Soutar et al. ${ }^{2}$ include the presence of a single area of bone destruction or soft tissue mass due to clonal plasma cells with histologically normal marrow aspirate and trephine, absent or low serum/urinary level M-component, and there is no either evidence of systemic impairment due to plasma cell

\section{Soung Min Kim}

Department of Oral and Maxillofacial Surgery, School of Dentistry, Seoul National University, 101 Daehak-ro, Jongno-gu, Seoul 03080, Korea

TEL: +82-2-2072-0213

E-mail:smin5@snu.ac.kr

ORCID: https://orcid.org/0000-0002-6916-0489

*These authors contributed equally to this work as first authors.

(c) This is an open-access article distributed under the terms of the Creative Commons Attribution Non-Commercial License (http://creativecommons.org/ licenses/by-nc/4.0/), which permits unrestricted non-commercial use, distribution, and reproduction in any medium, provided the original work is properly cited.

Copyright (C) 2021 The Korean Association of Oral and Maxillofacial Surgeons. dyscrasia (anemia, hypercalcemia, or kidney dysfunction) or additional lesions on skeletal survey and magnetic resonance imaging scan of the spine. The predominant site of SBP is the axial skeleton, especially vertebrae, whereas SEP is usually detected in the head and neck region. SEP symptoms are usually caused by expansion and invasion of plasma cell mass, including obstruction of the upper respiratory tract, swelling, and bony pain. Despite the fact that SEPs arise predominantly from the upper airway tract submucosal tissue and $80 \%$ of these arise from the nasal cavity and paranasal sinus, the related SP of maxillary sinus (SPMS) has rarely been reported. SPMS has been reported as an isolated disease or the first manifestation following multiple myeloma (MM) $)^{3,4}$.

Various protocols have been utilized for SPMS treatment, including surgery, radiotherapy, and chemotherapy ${ }^{5}$. Due to the rarity of this tumor, consensus on an optimal treatment strategy is lacking. In addition, despite early diagnostic and treatment, there are high incidences of locally recurrent and especially disseminated systemic MM, often months or even years later. Herein, a very rare case of SPMS is reported with the discussion regarding SPMS presence and current trends in treatment strategy.

\section{Case Report}

This article was approved by the Institutional Review Board of Seoul National University (S-D20200010). A 37-year-old male was referred to the Seoul National University Dental 

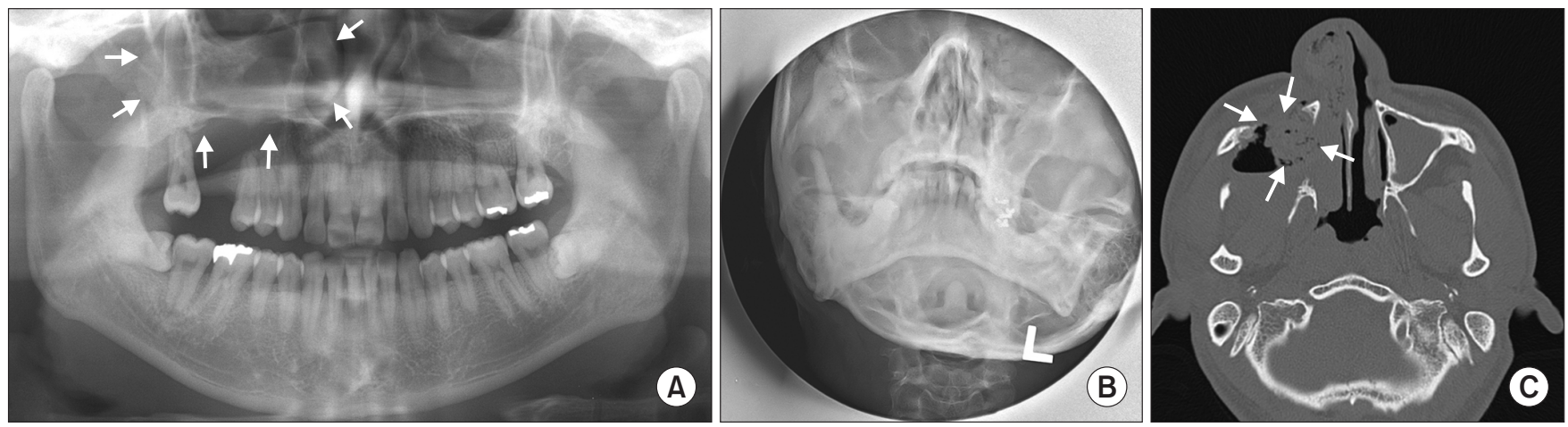

Fig. 1. Preoperative panoramic (A), Waters' view (B), and computed tomography axial view (C) revealed a mass within the right maxillary sinus (arrows). The mass destroyed the anterior, medial, and posterior walls of the sinus and extended into the right nasal cavity. Truc Thi Hoang Nguyen et al: Solitary plasmacytoma in the maxillary sinus: 10-year follow-up. J Korean Assoc Oral Maxillofac Surg 2021
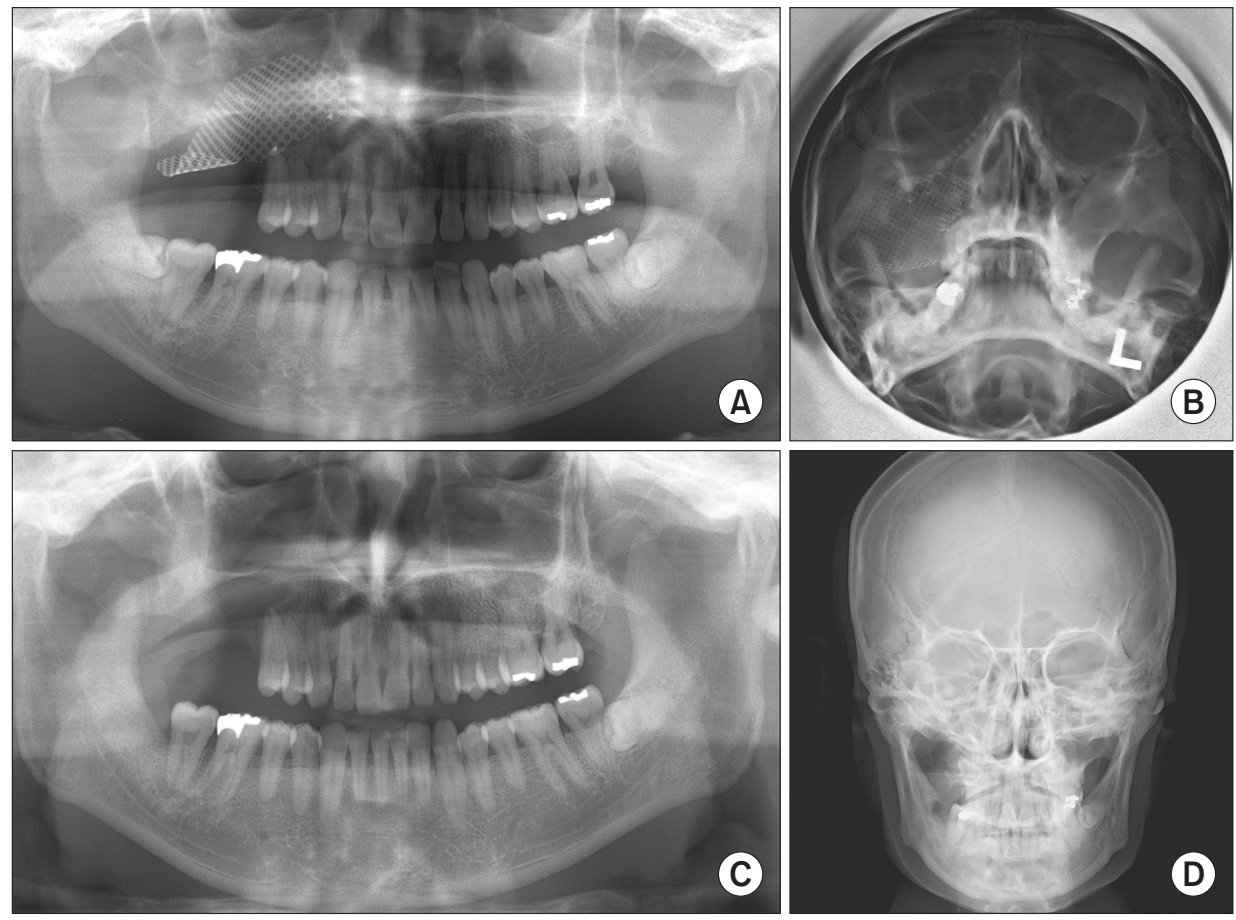

Fig. 2. Panoramic $(A)$ and Waters' view (B) one month after recession surgery. Panoramic (C) and plain skull (D) views after mesh removal surgery.

Truc Thi Hoang Nguyen et al: Solitary plasmacytoma in the maxillary sinus: 10-year follow-up. J Korean Assoc Oral Maxillofac Surg 2021

Hospital with a chief complaint of fullness and obstruction of the right nasal sinus, pain in the right maxillary region, and mobility in \#17 tooth. The patient was prescribed an oral antibiotic, oral antihistamine, and nasal antihistamine sprays; however, the swelling did not respond to the therapy. On extraoral examination, the nasal septum was found deviated to the right due to the enlargement of the right inferior turbinate. There was no palpable cervical node. The intraoral examination revealed a slight swelling in the vestibular space of the right maxilla. At the alveolar bone region of the extracted \#16 tooth, communication between oral and maxillary sinus was observed. The rest of the physical examination was normal.

The panoramic and Waters' views showed a large mass within the right maxillary sinus. Computed tomography (CT) showed thickening of the mucosa and opacification with the destruction of the medial and posterior wall of the right maxillary sinus. Skeletal radiographic examination of the mandible and other parts of the body was normal.(Fig. 1) Bone marrow biopsy revealed a normal presentation of all three hematopoietic lines. Urine analysis results were negative for albumin and Bence Jones protein. Immunoelectrophoresis did not show paraproteinemia.

The patient underwent mass excision and reconstruction of the sinus base with a metal mesh.(Fig. 2) Histopathological examination showed fibrotic tissue infiltrated by plasma cells with various stages of maturity and atypia. Plasma cells had enlarged nuclei, high nucleus-to-cytoplasm ratio, and typically "clock face" heterochromatin arrangement.(Fig. 3) Biopsy 


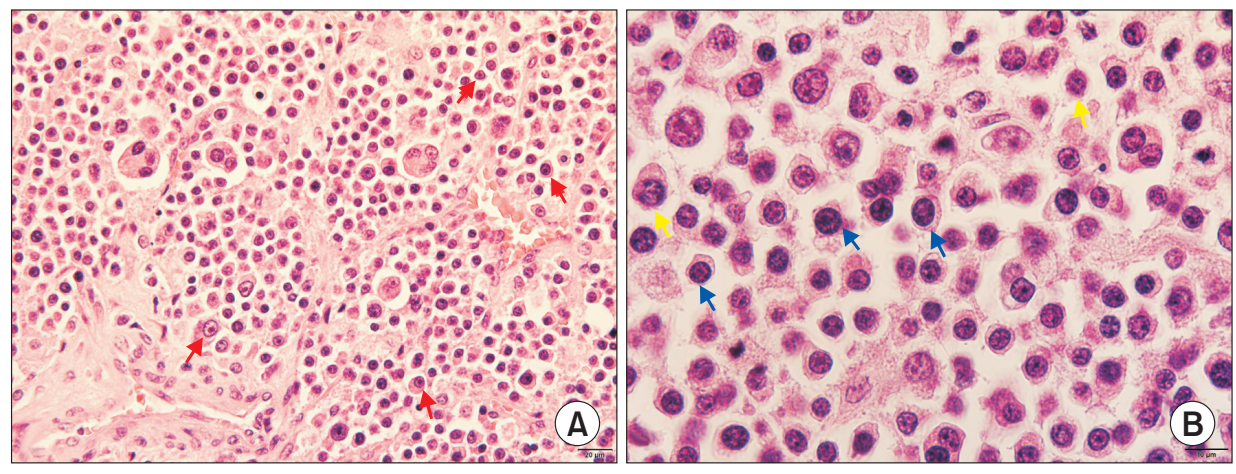

Fig. 3. Light microscopy photomicrograph of H\&E staining of maxillary sinus plasmacytoma. A. Atypical plasma cells with typical cartwheel or clock face heterochromatin arrangement (red arrows) (×200). B. Plasma cells show various stages of maturity with enlarged nuclei and high nucleus-to-cytoplasm ratio (blue arrows) and mitosis (yellow arrows) ( $\times 400$ ).

Truc Thi Hoang Nguyen et al: Solitary plasmacytoma in the maxillary sinus: 10-year follow-up. J Korean Assoc Oral Maxillofac Surg 2021

specimens were stained by kappa lambda $(\kappa: \lambda)$ light chain monoclonal antibodies, CD45, S-100, UCHL-1, L26, and CD3. Only CD45 staining showed a positive result and the other markers were negative. Based on clinical, radiographic, and laboratory findings, the lesion was diagnosed as SPMS.

Surgical treatment was followed by localized radiotherapy with a radiation dose of $50 \mathrm{~Gy}$ in 25 fractions. Three months after excision surgery, the metal mesh was removed, and biopsy of the maxillary sinus was repeated. There was no evidence of residual tumor in maxillary buccal mucosa and maxillary sinus. After the removal of the mass, and a full recovering after metal mesh removal, the patient reported the disappearing of the obstruction felling and the pain, which was a huge release and it improved patient's anxiety condition and health-related quality of life a lot. After 10 years of follow-up, the patient was alive and in disease remission with no evidence of local recurrence or disseminated systemic $\mathrm{MM}$

\section{Discussion}

SP is characterized by a discrete mass of neoplastic monoclonal plasma cells, without evidence of systemic myeloma symptoms $^{6}$. SPMS is a rare manifestation of nonspecific initial symptoms. An SPMS mass could also have a similar presentation on radiographic examination to other malignant neoplasms in the maxillary sinus. The reported symptoms are sinus fullness, epistaxis, and usually painless unless there is secondary infection ${ }^{7,8}$. These symptoms usually appear late, when the tumor growth is large and the adjacent structure is invaded.

When plasma cell infiltrates in the localized mass is observed, a specific clinicopathological differential diagnosis between MM and SP is important because treatment and prognosis differ. Bone marrow aspiration and trephine biopsy are recommended for all patients with suspected SP. Other examinations to exclude MM include radiological skeletal survey, serum and urine electrophoresis. The $\kappa: \lambda$ ratio examination should be performed to determine the proportion of monoclonal cells ${ }^{9}$. There are two types of light chains in human immunoglobulin molecules, $\kappa$ chains, and $\lambda$ chains. Serum free light chains (sFLCs) are the excessively produced light chains that do not bind to heavy chains to form immunoglobulin molecules and circulate in the bloodstream. In plasma cell neoplasms, the malignant plasma cells produce an excess of M-protein, including FLCs. When the production of sFLCs exceeds the reabsorptive capacity of the kidney, FLC molecules can be detected in the urine ${ }^{10}$. Normal individuals have a relatively consistent balance between $\kappa$ and $\lambda$ levels ( $\kappa: \lambda$ ratio). FLCs can be detected using electrophoresis or immunoelectrophoresis of serum and urine and be considered as an important diagnostic marker for $\mathrm{MM}^{10}$.

Histopathological examination of plasmacytomas shows diffuse proliferation of plasma cells with various stages of maturation and atypia. The cells have enlarged, round nuclei and prominent nucleoli, and a high nucleus-to-cytoplasm ratio. Cartwheel or clock face is the traditional description for the typical heterochromatin arrangement of plasma cells. Different stages of mitosis also can be observed in histopathological examination. Differential diagnosis of plasmacytomas includes other benign and malignant conditions of the sinus such as carcinoma, non-Hodgkin's lymphoma, and malignant melanoma. Therefore, immunohistochemical staining can help in distinguishing plasma cell neoplasms and other malignant tumors. CD45 plays an important role in antigenmediated signaling and activation in lymphocytes, and there- 
fore it presents in the early stage of plasma cell neoplasm development. In the present case, the positive result of CD45 staining was helpful in making a definite diagnosis.

The optimal treatment protocol of SPMS remains controversial. Radiotherapy, chemotherapy, and surgical treatment can be utilized based on tumor size, invasiveness and metastasis state, as well as systemic conditions. In surgical treatment, a margin of at least $2 \mathrm{~cm}$ is recommended. Regarding radiotherapy, 40 to 50 Gy is the generally accepted total fractionated dose. In the case of the big tumor $(>5 \mathrm{~cm}$ in diameter), a higher dose of radiation may be necessary for effective treatment ${ }^{2}$. Prophylactic management of the nodes in the neck region has not been shown to result in a better prognosis so it is not currently recommended ${ }^{11}$. Radioresistant SEPs have been reported, and in these cases, adjuvant chemotherapy can be considered based on PET/CT results after initial radiotherapy ${ }^{9}$. As mentioned above, distinguishing original SP and SP secondary to MM, for which the systemic chemotherapy should be started as soon as possible, is also important. Long-term follow-up by an otolaryngologist and a hematologist is necessary to make an early diagnosis when the disease converts to disseminated MM.

SPB has been reported to have a higher probability of progression to MM compared with EMP; however, EMP patients are reported to rapidly progress to $\mathrm{MM}$, usually within 2 years ${ }^{12}$. Determining the origin of suspicious local plasma cell tumors is important because the treatment and prognosis are different. In the present case, the origin of the tumor was difficult to define due to the presence of bone destruction. Based on radiographic evidence, we determined the origin of this mass was from soft tissue rather than bone. Even though bone destruction of maxillary sinus medial and posterior walls was observed, orbital floor destruction and suborbital swelling or eye proptosis were not evident. In addition, the necrosis region was found in the center of the mass, indicating the mass originated from sinus mucosa. The treatment procedure included mass excision with a margin of $2 \mathrm{~cm}$ followed by radiotherapy.

Even though SPMS is a localized disease, lymph nodes involvement at initial diagnosis ranges from $5 \%$ to $20 \%$ of cases in the literature ${ }^{13,14}$. Metastasis of SPMS was also reported $^{15}$. In the present case, diffuse infiltration of adjacent structures, such as orbit, palate skull base, or skin was not evident. In addition, node or distance metastasis was not observed. These clinical manifestations indicate a good prognosis for this patient. The surgical local control in combination with localized radiotherapy produced satisfactory results with complete disease regression. The patient did not show any sign of local recurrence or systemic disseminated MM during 10 years of follow-up.

In conclusion, SPMS can be misdiagnosed as other malignant neoplasms in the early stage; therefore, the systemic examination may be dismissed. SPMS can be the first manifestation of MM; therefore, a thorough and meticulous systemic evaluation with more specific histological, phenotypical, and radiographical methods is essential to detect MM or other plasma cell neoplasms if present. Furthermore, because systemic myeloma progression can develop any time after the treatment of a localized lesion, the patient should be followed up for an extended period of time by both a related surgeon and hematologist.

\section{ORCID}

Truc Thi Hoang Nguyen, https://orcid.org/0000-00028667-6698

Mi Young Eo, https://orcid.org/0000-0001-7055-9924

Buyanbileg Sodnom-Ish, https://orcid.org/0000-00024239-1420

Yun Ju Cho, https://orcid.org/0000-0002-1818-5280

Soung Min Kim, https://orcid.org/0000-0002-6916-0489

\section{Authors' Contributions}

T.T.H.N. and M.Y.E. participated in data collection and wrote the manuscript. B.S-I. and Y.J.C. participated in the study design and helped to draft the manuscript. S.M.K. coordinated and approved the final manuscript.

\section{Acknowledgements}

This research was supported by Basic Science Research Program through the National Research Foundation of Korea (NRF) funded by the Ministry of Education (2017R1D1A1B03036054).

\section{Conflict of Interest}

No potential conflict of interest relevant to this article was reported. 


\section{References}

1. Dimopoulos MA, Moulopoulos LA, Maniatis A, Alexanian R. Solitary plasmacytoma of bone and asymptomatic multiple myeloma. Blood 2000;96:2037-44.

2. Soutar R, Lucraft H, Jackson G, Reece A, Bird J, Low E, et al.; Guidelines Working Group of the UK Myeloma Forum; British Committee for Standards in Haematology; British Society for Haematology. Guidelines on the diagnosis and management of solitary plasmacytoma of bone and solitary extramedullary plasmacytoma. Br J Haematol 2004;124:717-26. https://doi.org/10.1111/j.13652141.2004.04834.x

3. Ghazizadeh M, Alavi Amlashi H, Mehrparvar G. Radioresistant extramedullary plasmacytoma of the maxillary sinus: a case report and review article. Iran J Otorhinolaryngol 2015;27:313-8.

4. Jeyaraj P, Venkatesan M, Nijhawan VS. Solitary extramedullary plasmacytoma of the maxillary sinus, progressing to smoldering multiple myeloma with multifocal skeletal involvement, which resolved completely following chemotherapy alone. J Maxillofac Oral Surg 2016;15(Suppl 2):229-39. https://doi.org/10.1007/ s12663-015-0742-0

5. Cantone E, Di Lullo AM, Marano L, Guadagno E, Mansueto G, Capriglione P, et al. Strategy for the treatment and follow-up of sinonasal solitary extramedullary plasmacytoma: a case series. J Med Case Rep 2017;11:219. https://doi.org/10.1186/s13256-017$1382-4$

6. Kilciksiz S, Karakoyun-Celik O, Agaoglu FY, Haydaroglu A. A review for solitary plasmacytoma of bone and extramedullary plasmacytoma. ScientificWorldJournal 2012;2012:895765. https://doi. org/10.1100/2012/895765

7. Wax MK, Yun KJ, Omar RA. Extramedullary plasmacytomas of the head and neck. Otolaryngol Head Neck Surg 1993;109:877-85. https://doi.org/10.1177/019459989310900517

8. Tamada A, Araki T, Makimoto K, Takatsuki K. Plasmacytoma in nasal cavity and maxillary sinus. Arch Otorhinolaryngol 1982;237: 83-91. https://doi.org/10.1007/BF00453721
9. Caers J, Paiva B, Zamagni E, Leleu X, Bladé J, Kristinsson SY, et al. Diagnosis, treatment, and response assessment in solitary plasmacytoma: updated recommendations from a European Expert Panel. J Hematol Oncol 2018;11:10. https://doi.org/10.1186/ s13045-017-0549-1

10. Selvaratnam R, Cao J, Karger AB. Serum free light chain analysis. In: Linden MA, McKenna RW, eds. Plasma cell neoplasms: a morphologic, cytogenetic and immunophenotypic approach. Cham: Springer; 2016:25-42.

11. Strojan P, Soba E, Lamovec J, Munda A. Extramedullary plasmacytoma: clinical and histopathologic study. Int J Radiat Oncol Biol Phys 2002;53:692-701. https://doi.org/10.1016/s03603016(02)02780-3

12. Holland J, Trenkner DA, Wasserman TH, Fineberg B. Plasmacytoma. Treatment results and conversion to myeloma. Cancer 1992;69:1513-7. https://doi.org/10.1002/10970142(19920315)69:6<1513::aid-cncr2820690633>3.0.co;2-x

13. Kotner LM, Wang CC. Plasmacytoma of the upper air and food passages. Cancer 1972;30:414-8. https://doi.org/10.1002/10970142(197208)30:2<414::aid-cncr2820300217>3.0.co;2-z

14. Tsang RW, Campbell BA, Goda JS, Kelsey CR, Kirova YM, Parikh RR, et al. Radiation therapy for solitary plasmacytoma and multiple myeloma: guidelines from the International Lymphoma Radiation Oncology Group. Int J Radiat Oncol Biol Phys 2018;101:794-808. https://doi.org/10.1016/j.ijrobp.2018.05.009

15. Megat Shiraz MA, Jong YH, Primuharsa Putra SH. Extramedullary plasmacytoma in the maxillary sinus. Singapore Med J 2008;49: e310-1.

How to cite this article: Nguyen TTH, Eo MY, Sodnom-Ish

B, Cho YJ, Kim SM. Solitary plasmacytoma in the maxillary sinus: 10-year follow-up. J Korean Assoc Oral Maxillofac Surg 2021;47:471-475. https://doi.org/10.5125/jkaoms.2021.47.6.471 